BOOK BRIEFLY NOTED

\section{Direct Diagnosis in Radiology: Vascular Imaging}

K.-J. Wolf, Z. Grozdanovic, T. Albrecht, J. Heidenreich, A. Schilling, and F. Wacker, eds. Thieme; 2009, 295 pages, 250 illustrations, $\$ 59.95$.

Collowing the same format as previous volumes in this se-

ries, Direct Diagnosis in Radiology: Vascular Imaging, edited by Professor Karl-Jüergen Wolf, presents the reader with a wide range of vascular pathologies involving the brain, spine, head and neck, chest, abdomen, kidneys, and extremities. In the neurology area alone, there are 52 cases describing and illustrating abnormalities: developmental, congenital, acquired, ischemic, inflammatory, and tumoral. Each case is divided into bullet points in the following areas: definition, imaging signs, clinical aspects, differential diagnoses, tips/ pitfalls, and selected references. It is of interest to read through the different cases to solidify the appearance of each type of abnormality and to review the major concepts and differential point of the entities. The imaging is straightforward, and there is little problem in identifying the major features and establishing a diagnosis because the selected CT scans, MR images, and angiograms are of good quality. Nearly half the book (124 of 283 pages of cases) deals with cases in the realm of neurora-
Direct Diagnosis in Radiology Vascular Imaging

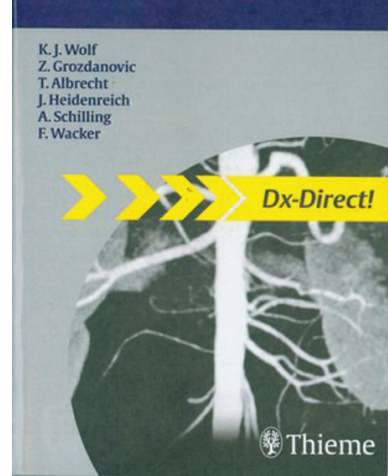
diology. I do not believe this soft-cover 295-page book would be primarily of interest to a neuroradiologist; nonetheless, it would fit nicely in a departmental library to add to the other books in the series.

DOI 10.3174/ajnr.A1686 